\title{
Adsorption of desflurane by the silica gel filters in breathing circuits: an in vitro study
}

\author{
Seok Young Song, Bo Reum Lim, and Taeha Ryu \\ Department of Anesthesiology and Pain Medicine, School of Medicine, Cathoilic University of Daegu, Daegu, Korea
}

Background: During general anesthesia, a heated breathing circuit (HBC) is used to replace the heat and moisture exchange function of the upper airway. One HBC uses an air dryer filter that employs silica gel (SG) as a desiccant. SG is capable of adsorbing many organic compounds. Therefore, we undertook an in vitro study of the adsorption of desflurane by SG filters.

Methods: An HBC was connected to an anesthesia machine, and a test lung was connected to the circuit. The test lung was mechanically ventilated with 2 or $4 \mathrm{~L} / \mathrm{min}$ of fresh gas flow, with and without the air dryer filter. Desflurane was administered at a 6 vol\% on the vaporizer dial setting. The experiment was repeated 15 times in each group. The end-tidal concentrations were measured during the experiments. The air dryer filter weights were measured before and after the experiments, and the times required to achieve the specific end-tidal desflurane concentrations were determined.

Results: Significant differences in the end-tidal concentrations of desflurane were observed between the control and filter groups $(\mathrm{P}<0.001)$. The filter weights increased significantly after the experiments $(\mathrm{P}<0.001)$. The times required to achieve the same end-tidal desflurane concentrations were different with the application of the air dryer filter $(\mathrm{P}<0.001)$. Conclusions: The adsorption of desflurane with the use of an air dryer filter was verified in this in vitro study. Careful attention is needed when using air dryer gel filters during general anesthesia.

Key Words: Air filters, Adsorption, Desflurane, Desiccation, Silica gel.

Corresponding author: Taeha Ryu, M.D.

Department of Anesthesiology and Pain Medicine, School of Medicine, Cathoilic University of Daegu, 33, Duryugongwon-ro 17-gil, Nam-gu, Daegu 705-718, Korea

Tel: 82-53-650-4785, Fax: 82-53-650-4517

E-mail: anesryu@cu.ac.kr

It was presented The 90th Annual Scientific Meeting of the Korean Society of Anesthesiologists, November 2013, Kangwon Land Convention Center, Jeongseon, Korea.

Received: May 19, 2014.

Revised: August 23, 2014.

Accepted: September 23, 2014.

Korean J Anesthesiol 2015 June 68(3): 274-280

http://dx.doi.org/10.4097/kjae.2015.68.3.274

\section{Introduction}

The upper airway warms and humidifies the inspired air to prevent drying of the secretions in the lower airway tracts, plugging and mucosal injury. Bypassing the upper airway by endotracheal intubation during general anesthesia can lead to the loss of the air conditioning function of the upper airway. Heated breathing circuits (HBCs) are used to replace upper airway function during general anesthesia.

The potential hazards of HBCs include thermal lung injury, nosocomial infection, increased likelihood of circuit disconnection, increased airway resistance from excess water condensation in the breathing circuit, and interference with flow meter function. Therefore, one heated breathing circuit (Heated Circuit, ACE Medical, Seoul, Korea) uses an air dryer filter to remove water vapor from exhaled air, and it prevents water vapor condensation in the anesthesia machine. This HBC air dryer

(c) This is an open-access article distributed under the terms of the Creative Commons Attribution Non-Commercial License (http://creativecommons.org/ licenses/by-nc/4.0/), which permits unrestricted non-commercial use, distribution, and reproduction in any medium, provided the original work is properly cited. 
filter uses silica gel (SG) as a desiccant.

In a recent case report, a misconnected air dryer filter led to an insufficient desflurane concentration during general anesthesia [1], and the authors proposed that the SG in the air dryer filter had adsorbed desflurane.

However, no studies about SG adsorption of desflurane are available, and only one experimental study of SG's adsorption of isoflurane exists [2]. Therefore, we undertook an in vitro study of the adsorption of desflurane by HBC air dryer filters.

\section{Materials and Methods}

The in vitro experimental study was performed using an anesthesia machine (Aestiva S/5 anesthesia delivery system, GE Datex-Ohmeda, Munich, Germany) that is currently used in our hospital for general anesthesia.

A schematic diagram of this experiment is shown in Fig. 1. The HBC (Heated Circuit, ACE Medical, Seoul, Korea) was connected to the anesthesia machine, and a test lung (Maquet, $\mathrm{Mu}$ nich, Germany) was connected to the circuit. The test lung was mechanically ventilated using the volume control mode (tidal volume $500 \mathrm{ml}$, respiratory rate 10 breaths/min, IE ratio $1: 2$ ) with and without the air dryer filter. An air dryer filter was attached to the expiratory limb of the HBC. Desflurane was administered at a $6 \mathrm{vol} \%$ on the vaporizer dial setting with a fresh gas flow (FGF) of $100 \% \mathrm{O}_{2}$.

The experiments were conducted with two FGF rates $(2 \mathrm{~L} / \mathrm{min}$ and $4 \mathrm{~L} / \mathrm{min}$ ) in two subgroups based on the application of the air dryer filter. Fifteen trials were performed for each subgroup (a total of 4 groups: Control 2L, Filter 2L, Control 4L, and Filter
$4 \mathrm{~L})$; a total of 60 trials were conducted.

An external gas analyzer (IntelliVue Anesthetic Gas Modules G5, Philips, Boblingen, Germany) was used for anesthetic concentration measurements. A gas sample of $200 \mathrm{ml} / \mathrm{min}$ was obtained from the Y-piece of the circuit between the circuits and the test lung. The end-tidal concentrations of desflurane were measured every 12 seconds for 30 minutes. The end-tidal desflurane concentrations after administration every 5 minutes were compared with the applications of the air dryer filter.

To minimize the effects of remnant desflurane from each previous test, a washout procedure was performed for the next experiment as follows. A new breathing circuit was attached to the anesthesia machine, and a new test lung was connected to the Y-piece of the circuit. The circuit and anesthetic machine were flushed with oxygen using mechanical ventilation for a minimum of 40 minutes. Following the washout procedure, the carbon dioxide absorbent was renewed, and the new HBC was connected to the anesthesia machine for the next experiment.

The experiments were conducted at an operating room temperature of $21^{\circ} \mathrm{C}$. To minimize the influences of heat and humidity on adsorption, we did not heat the circuit or add water to it.

To verify the adsorption of desflurane, the filter weights were measured before and after each experiment. To assess the influence of the air dryer filter on the desflurane wash-in times, the times to achieve 90 and 75\% wash-in were determined. The endtidal wash-in concentrations were 5.4 and 4.5 vol\%, respectively.

All data are presented as means \pm standard deviations. Normality testing of the measured variables was performed by both visual inspection of the Q-Q plots and the application of the Shapiro-Wilk test. No deviations from normality were detected,

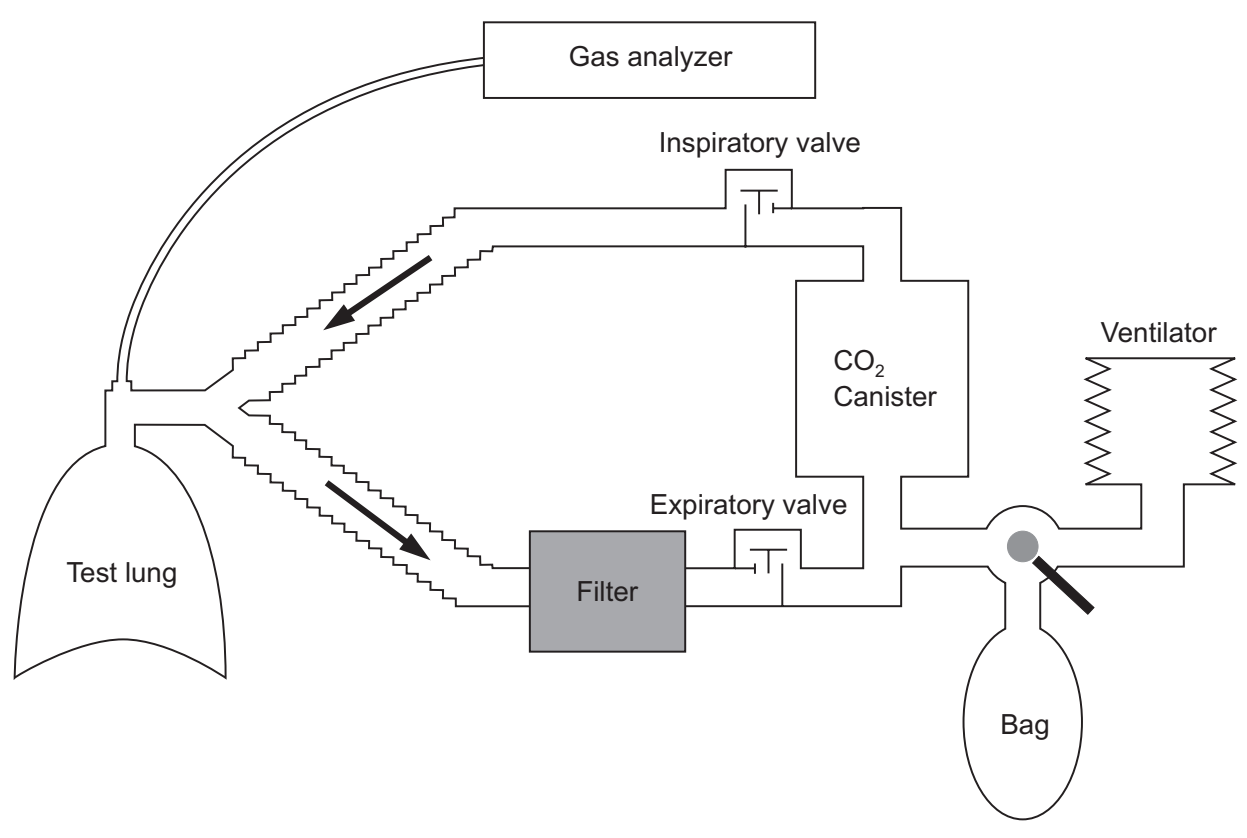

Fig. 1. Schematic diagram for the experiment. A heated breathing circuit (HBC) was connected to an anesthesia machine, and a test lung was connected to the Y-piece of the circuit. A silica gel filter was placed at the expiratory limb of the circuit. The gas sampling tube for the external gas analyzer was connected to the Y-piece between the $\mathrm{HBC}$ and the test lung. 
justifying the use of parametric testing.

Repeated-measured analysis of variance was used to detect differences in the end-tidal desflurane concentrations with application of the air dryer filter with each FGF. The significance level was set at 0.05 , and adjustments were made if a violation of sphericity was identified (a Huynh-Feldt adjustment if the sphericity estimate was $>0.75$ or Greenhouse-Geisser otherwise). The paired t-test was used to compare the filter weights before and after the experiments in each FGF. Student's t-test was used to compare the weight changes between 2 and $4 \mathrm{~L} / \mathrm{min}$ FGF and for comparing the wash-in times to achieve the specific concentrations of each FGF. Statistical analyses were performed using IBM SPSS Statistics version 19.0.0 (IBM Corp., Armonk, NY, USA). P values less than 0.05 were considered significant.

\section{Results}

Significant differences in end-tidal desflurane concentrations were observed depending on the application of the air dryer filter with both FGFs ( $\mathrm{P}<0.001$, Fig. 2$)$, and significant differences were also observed in the end-tidal desflurane concentrations depending on the FGF ( $\mathrm{P}<0.001$, Fig. 2).

The air dryer filter weights increased significantly from $94.36 \pm$ $1.15 \mathrm{~g}$ before the experiments to $104.3 \pm 1.07 \mathrm{~g}$ after the experiments with $4 \mathrm{~L} / \mathrm{min}$ of FGF and from $94.76 \pm 1.00 \mathrm{~g}$ before the

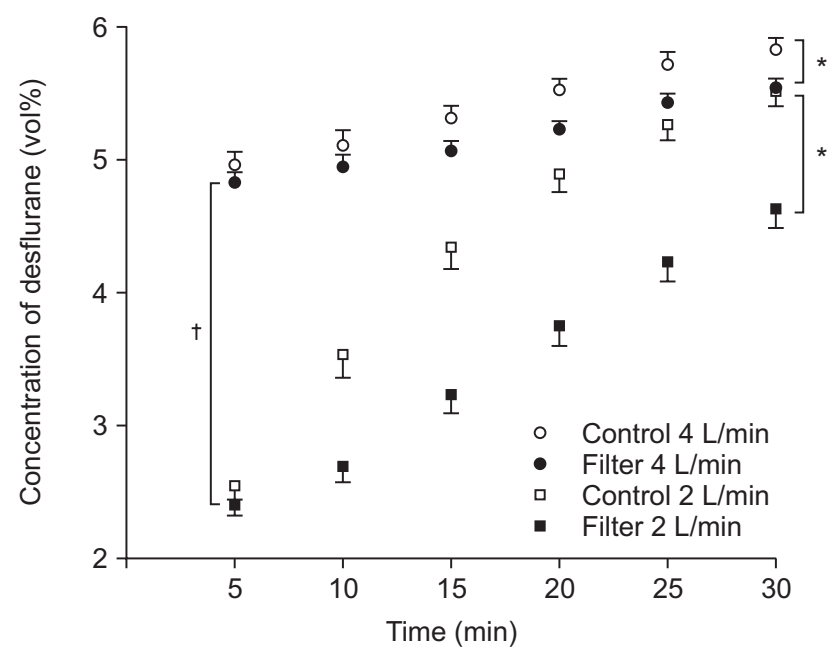

Fig. 2. Comparison of the end-tidal desflurane concentration time curves. The experiments were conducted with FGFs of $4 \mathrm{~L} / \mathrm{min}$ (circles) and $2 \mathrm{~L} / \mathrm{min}$ (squares) in two subgroups based on the application of the air dryer filter (open symbols: control group without silica gel filter; filled symbol: experimental group with silica gel filter). *Significant differences in the end-tidal desflurane concentrations were observed between the control and filter groups with both $4 \mathrm{~L} / \mathrm{min}$ (open vs. filled circles) and $2 \mathrm{~L} / \mathrm{min}$ fresh gas flows (open vs. filled squares) $(\mathrm{P}<0.001)$.

${ }^{\dagger}$ The end-tidal desflurane concentrations with air dryer filters were significantly different between $4 \mathrm{~L} / \mathrm{min}$ (filled circles) and $2 \mathrm{~L} / \mathrm{min}$ of fresh gas flow (filled squares) $(\mathrm{P}<0.001)$. experiments to $103.61 \pm 1.09 \mathrm{~g}$ after the experiments with $2 \mathrm{~L} / \mathrm{min}$ of FGF $(\mathrm{P}<0.001$, Table 1$)$. The changes in filter weights after the experiments were significantly different between the two FGFs: $9.94 \pm 0.54 \mathrm{~g}$ with $4 \mathrm{~L} / \mathrm{min}$ of FGF and $8.85 \pm 0.56 \mathrm{~g}$ with $2 \mathrm{~L} / \mathrm{min}(\mathrm{P}<0.001$, Table 1$)$.

With $4 \mathrm{~L} / \mathrm{min}$ FGF, the times required to achieve $90 \%$ desflurane wash-in were $16.85 \pm 1.88 \mathrm{~min}$ without the filter and 24.09 \pm 2.13 min with the filter; significant differences in the times required to achieve $90 \%$ desflurane wash-ins were observed between the filter and the control groups (Fig. 3). With a $2 \mathrm{~L} /$ min FGF, the time required to achieve $90 \%$ wash-in of desflurane without a filter was $27.12 \pm 2.31 \mathrm{~min}$. However, the time to achieve $90 \%$ desflurane wash-in with $2 \mathrm{~L} / \mathrm{min}$ FGF with the filter could not be measured (Fig. 3) because the desflurane concentration after the experiments (30 minutes subsequent) was

Table 1. Filter Weights before and after the Experiments

\begin{tabular}{|c|c|c|c|}
\hline & \multicolumn{2}{|c|}{ Air dryer filter weights } & \multirow{2}{*}{$\Delta$ Weight $^{\dagger}(\mathrm{g})$} \\
\hline & Before experiments (g) & After experiments (g) & \\
\hline FGF $4 \mathrm{~L} / \mathrm{min}$ & $94.36 \pm 1.15$ & $104.30 \pm 1.07^{*}$ & $9.94 \pm 0.54$ \\
\hline FGF $2 \mathrm{~L} / \mathrm{min}$ & $94.76 \pm 1.00$ & $103.61 \pm 1.09 *$ & $8.85 \pm 0.56$ \\
\hline
\end{tabular}

Values are mean $\pm \mathrm{SD}$. *The air dryer filter weights changed significantly with both fresh gas flows compared with the pre-experiment weights $(\mathrm{P}$ $<0.001)$. ${ }^{\dagger}$ The filter weight changes were greater with $4 \mathrm{~L} / \mathrm{min}$ of fresh gas flow compared with the $2 \mathrm{~L} / \mathrm{min}$ FGF $(\mathrm{P}<0.001)$. FGF: fresh gas flow. $\Delta$ Weight: the differences of filter weights between before and after experiments.

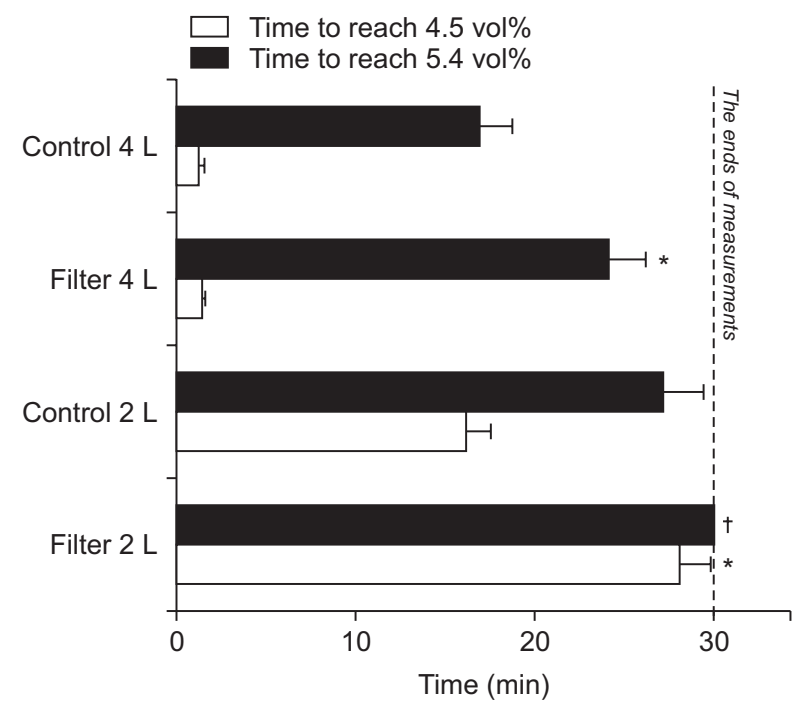

Fig. 3. Times to achieve specific end-tidal desflurane concentrations. The dashed line indicates when the measurements ended. *More time was required to achieve the specific end-tidal desflurane concentrations with air dryer filters compared with the control group $(\mathrm{P}<0.001)$.

${ }^{\dagger}$ The end-tidal desflurane concentration did not reach 5.4 vol\% after 30 minutes, and therefore these time data could not be obtained. 
$4.63 \pm 0.14$ vol $\%$; i.e., the concentration did not reach 5.4 vol\% during the experiments (Fig. 3).

With a $4 \mathrm{~L} / \mathrm{min}$ FGF, the times required to achieve $75 \%$ desflurane wash-in were $1.21 \pm 0.23 \mathrm{~min}$ without the filter and $1.41 \pm$ 0.13 min with it (Fig. 3). Statistical significance was not observed. With a $2 \mathrm{~L} / \mathrm{min}$ FGF, the times required to achieve $75 \%$ desflurane wash-in were $16.24 \pm 1.27 \mathrm{~min}$ without the filter and 28.12 \pm 1.71 min with it. Significant differences in the times to reach $75 \%$ desflurane wash-in were observed between the filter and the control groups with $2 \mathrm{~L} / \mathrm{min}$ of FGF (Fig. 3).

\section{Discussion}

Significant differences were observed in the end-tidal desflurane concentrations based on the application of the air dryer filter irrespective of the amount of the FGF. The time required to reach the same end-tidal desflurane concentration was longer with the application of the air dryer filter. The filter weights increased significantly after the experiments. Therefore, we verified that SG was able to adsorb desflurane vapor in this in vitro study.

SG is an adsorbent that is most frequently known as a desiccant. SG is often considered to absorb moisture, but it removes moisture by adsorption rather than absorption in a strict sense.

Adsorption is similar to absorption, but the processes differ. Adsorption is the adhesion of molecules to the surface of the adsorbent, whereas absorption is the assimilation of substances within the bulk of the absorbents. Thus, in adsorption, the concentration of adsorbate is higher at the adsorbent surface. In contrast, in absorption, the absorbate molecules are uniformly distributed, and the absorbate concentration is the same throughout the bulk of the absorbents [3].

SG is a highly porous form of silicon dioxide. The adsorptive property of SG results from this high porosity [4]. The internal surface area of SG is approximately $700-800 \mathrm{~m}^{2} / \mathrm{g}$, and it is generally said that the internal surface of a teaspoonful of SG is comparable to the area of a football field [5].

Various adsorbents have been used in medicine. Activated charcoal is the most often used adsorbent in medical applications. It is used for the acute management of poisoning caused by drugs or chemicals [6-8]. Activated charcoal has been known to adsorb volatile anesthetics, such as ether, trichloroethylene, halothane, methoxyflurane, isoflurane and sevoflurane $[9,10]$. It is used to adsorb volatile anesthetics in anesthesia research and practice. As an adsorber, activated charcoal efficiently prevents atmospheric isoflurane contamination during isoflurane sedation [11]. Recently, the attachment of a charcoal filter to the anesthesia circuit shortened the extubation time and the time to eye opening without adverse effects [10]. Activated charcoal has been considered and tested in the preparation of anesthesia machines for patients with malignant hyperthermia [12-14]. The AnaConDa ${ }^{\circledR}$ filter, which has charcoal as the adsorber, was introduced to reduce the consumption of volatile anesthetics and prevent air pollution $[15,16]$.

Another adsorbent, zeolite, can also adsorb volatile anesthetics. In an animal study, isoflurane consumption was reduced by approximately $70 \%$ using a zeolite reflection filter [17]. Zeolite effectively removes isoflurane from exhaled gases [18]. Zelolites allow for the near-complete desorption of adsorbed desflurane, and the possibility of recycling the scavenged volatile anesthetics has been proposed [19].

However, studies regarding the adsorption of volatile anesthetics by $S G$ are rare.

SG is commonly used as a dehydrating agent. Unlike other adsorbents, SG has been generally regarded as a desiccant rather than an adsorbent. However, it has not only a dehydrating but also an adsorptive function. Dehydration is one of SG's adsorption functions. Owing to the insufficient understanding of the adsorption property of SG, it would appear that SG is being used as a desiccant in heated circuits. Although few reports are available on SG's adsorption of volatile anesthetics, it can be easily inferred from the adsorptive property of adsorbents that SG is capable of adsorbing volatile anesthetics.

SG has the ability to adsorb many organic compounds, such as ethers, ketones, aromatic hydrocarbons, and halogenated hydrocarbons. The adsorption of isoflurane by SG has already been reported [2]. Commonly administered volatile anesthetics, such as isoflurane, halothane, sevoflurane and desflurane, are chemically 'halogenated hydrocarbons' that are all closely related. Desflurane (2,2,2-trifluoro-1-fluoroethyl-difluoromethyl ether) is a highly fluorinated methyl ethyl ether. The structure of desflurane is particularly similar to isoflurane with the exception of the substitution of a fluorine atom for isoflurane's chlorine atom [20]. Studies on SG's adsorption of desflurane are not available, but this adsorption is easily anticipated given the structure of desflurane.

Several papers have described the absorption of volatile anesthetics by SG. SG has been used to collect halothane in the air of operating rooms to evaluate halothane exposure [21]. Lumb and Landon [2] reported that approximately $35 \mathrm{ml}$ of dry SG absorbed 1,000 and 1,085 $\mathrm{ml}$ of isoflurane vapor, amounts equal to 29 and 31 times its own volume, respectively. In a recent case report, the adsorption of desflurane by a misconnected air dryer filter led to insufficient desflurane concentrations during general anesthesia [1].

The majority of modern anesthetic machines are equipped with a circle rebreathing system. The inspired gas contains fresh gas and rebreathed gas. In high FGFs, the inspired anesthetic concentration is more influenced by the anesthetic gas fraction of FGF. Conversely, the anesthetic gas fraction of rebreathing has a 
greater effect on the inspired anesthetic concentration at low FGFs.

The air dryer filter of the HBC should be placed in the expiratory limb of the circuit. The expired anesthetic gas fraction is adsorbed by the SG filter in the expiratory limb of the circuit, and the adsorption of anesthetic gases by SG filters affects the anesthetic gas fraction of rebreathed gases. Therefore, the impact of anesthetic gas adsorption is higher at a low FGF. These rebreathing properties of are supported by the times that we found necessary to achieve specific end-tidal desflurane concentrations in this experiment. The anesthetic gas concentrations of the circuit are more influenced by the anesthetic gas fraction of FGF at a $4 \mathrm{~L} / \mathrm{min}$ FGF. Conversely, the anesthetic concentrations are more influenced by rebreathed gas with a $2 \mathrm{~L} / \mathrm{min}$ of FGF.

At an FGF of $4 \mathrm{~L} / \mathrm{min}$, a statistically significant difference was not observed with the times required to achieve $75 \%$ washins between the filter and the control groups, but significant differences in the times required to achieve $90 \%$ wash-ins were observed. Initially, the end-tidal anesthetic concentration was the same for the control and filter groups were because the endtidal anesthetic concentration is influenced by the relatively high FGF. Later, the end-tidal anesthetic concentration between the control and filter groups was significantly different due to the adsorption of rebreathed anesthetic gas. At an FGF of $2 \mathrm{~L} / \mathrm{min}$, a significant difference was observed with $75 \%$ wash-in times between the filter and the control groups. The end-tidal anesthetic concentration was affected by the filter from the beginning because the anesthetic gas fraction of rebreathing has a greater effect on the inspired anesthetic concentration at an FGF of $2 \mathrm{~L} /$ min. Therefore, the impact of anesthetic gas adsorption by the SG filter is greater at a low FGF.

In this study, the filter weights increased to $9.94 \pm 0.54 \mathrm{~g}$ after 30 min of experiments using a $4 \mathrm{~L} / \mathrm{min}$ FGF. The density of desflurane was $1.45 \mathrm{~g} / \mathrm{ml}$, and the weight of the adsorbed desflurane was $9.94 \mathrm{~g}$. Therefore, approximately $6.86 \mathrm{ml}$ were adsorbed as a liquid.

Assuming that desflurane is an ideal gas, the equivalent vapor volume of adsorbed liquid desflurane can be easily calculated from the desflurane density and the amounts of adsorbed desflurane using Avogadro's hypothesis and Charles's law. Avogadro's hypothesis states that 1 mole of a liquid when vaporized occupies $22.4 \mathrm{~L}$ at standard temperature and pressure (STP, $0^{\circ} \mathrm{C}$ and $760 \mathrm{mmHg}$ ). The molecular weight of desflurane is 168 , and therefore, the weight of 1 mole of desflurane is $168 \mathrm{~g}$; in addition, when vaporized, 1 mole of desflurane occupies $22.4 \mathrm{~L}$ at STP. One gram of adsorbed desflurane is approximately 133.3 $\mathrm{ml}$ of desflurane vapor at STP. Therefore, the vapor volume of adsorbed desflurane was $1.33 \mathrm{~L}$ at STP and $1.43 \mathrm{~L}$ at $21^{\circ} \mathrm{C}$ and standard pressure, as calculated by Charles's law.

Using a $2 \mathrm{~L} / \mathrm{min} \mathrm{FGF}$, the filter weights increased to $8.85 \pm$ $0.54 \mathrm{~g}$ after $30 \mathrm{~min}$ of experiments. The volume of the adsorbed desflurane liquid was approximately $6.10 \mathrm{ml}$ using the calculations mentioned above. The volume of desflurane vapor is $1.18 \mathrm{~L}$ at STP and $1.27 \mathrm{~L}$ at $21^{\circ} \mathrm{C}$ and standard pressure.

The amount of delivered desflurane can be roughly calculated using the FGF and the vaporizer dial setting. Using a $4 \mathrm{~L} / \mathrm{min}$ FGF, 6 vol\% desflurane was delivered for $30 \mathrm{~min}$, and therefore, the delivered desflurane vapor was 7.2 L. Consequently, approximately $19.9 \%$ of the delivered desflurane (1.43 L of desflurane vapor) was absorbed by the air dryer filter at an FGF of $4 \mathrm{~L} / \mathrm{min}$.

With a $2 \mathrm{~L} / \mathrm{min}$ FGF, the amount of delivered desflurane vapor was $3.6 \mathrm{~L}$ according to the calculations described above. Therefore, approximately $35.3 \%$ of the delivered desflurane (1.27 $\mathrm{L}$ desflurane vapor) was absorbed by the air dryer filter at an FGF of $2 \mathrm{~L} / \mathrm{min}$.

The proportion of desflurane adsorption depends on the FGF; a greater proportion of desflurane is adsorbed at lower FGFs than at higher flows. Therefore, it can be said that desflurane wash-in is more affected at lower FGFs.

In the experiment by Lumb and Landon, adsorption of isoflurane did not occur when the SG was fully saturated with water vapor [21], and the adsorbed isoflurane was displaced with a more polar compound, such as water. In clinical situations, the adsorption of anesthetics may be affected by water from the patient's expiration and the added water of the HBC. However, air dryer filters are not fully hydrated during general anesthesia. Unless the air dryer filter is fully hydrated, adsorption may continue during general anesthesia. In this experimental study, we did not add water or heat to the circuit, and the effect of hydrated SG on the adsorption was not studied.

All of the halogenated volatile anesthetics are recognized greenhouse gases [22,23].

Two recent papers provide the global warming potentials (GWP) of volatile anesthetics [22,23]. GWP is a value for comparing the heat-trap abilities of greenhouse gases with that of carbon dioxide. Ryan and Nielsen [22] provided the GWP20 values for volatile anesthetics, and Sulbaek Andersen et al. [23] provided the GWP100 values for volatile anesthetics. In particular, desflurane has the greatest effect on the environment among the volatile anesthetics.

The consumption of volatile anesthetics may be increased if an SG filter is used during general anesthesia. The adsorption process is reversible, and therefore, desorption of the volatile anesthetics from the disposed SG filters could adversely affect the environment if the air dryer filter is not processed before disposal.

This study has some limitations. First, because we used only one anesthesia machine in our experiments, rebound effects from silicone and rubber components could have occurred despite the time spent washing out the anesthesia machine [12]. Because of the rebound effect, an increased washout time might 
be required for precise experiments.

Second, the environmental temperature and humidity was not measured. Although no water was added to the circuit, environmental humidity could affect the weight changes of filters.

Third, true anesthetic practices are not identical to the conditions used in this experiment. HBCs are used with heat and added water in clinical anesthetic practices, but heat and water were not added in this experiment. Exhaled water vapor is present during general anesthesia, but it was not present in this experiment.

Furthermore, volatile anesthetic absorptions by patients were not considered in this experiment. In clinical situations, the inhalation anesthetic wash-in is affected by the uptake of anesthetics by the blood in the patient's lungs. Therefore, the inhalation anesthetic wash-in differs according to the patient's disease states, especially conditions such as cardiac disease or pulmonary disease. In this experiment, there was very little uptake by the test lung. This experiment was not identical to clinical anesthetic practice. Therefore, additional studies regarding the adsorption of anesthetics by air dryer filters during general anesthesia should be performed.

In conclusion, silica gel is adsorbent, and SG filters can adsorb volatile anesthetics. In this experiment, desflurane adsorption using an SG filter was verified by the increased filter weights. The adsorption of desflurane using an SG filter also resulted in lower end-tidal desflurane concentrations and longer times to reach specific desflurane concentrations. Therefore, careful attention should be paid when using SG filters during general anesthesia. Additional study will be required to verify whether the adsorption of desflurane by SG filters affects anesthetic concentrations in clinical situations.

\section{References}

1. Lee JE, Kwon OD, Kim JC, Ryu TH. The adsorption of inspired desflurane due to incorrectly mounted air dryer filter of the heated humidifier: A case report. Anesth Pain Med 2013; 8: 141-4.

2. Lumb AB, Landon MJ. Absorption of isoflurane by silica gel. Br J Anaesth 1991; 67: 106-7.

3. Dąbrowski A. Adsorption—from theory to practice. Adv Colloid Interface Sci 2001; 93: 135-224.

4. Saha BB, Akisawa A, Kashiwagi T. Solar/waste heat driven two-stage adsorption chiller: the prototype. Renew Energy 2001; 23: 93-101.

5. Weintraub S. Demystifying Silica Gel. In: Objects Specialty Group Postprints: Volume 9, 2002. Washington DC, American Institute for Conservation, 2002. pp 169-94.

6. Neuvonen PJ, Olkkola KT. Oral activated charcoal in the treatment of intoxications. Role of single and repeated doses. Med Toxicol Adverse Drug Exp 1988; 3: 33-58.

7. McLuckie A, Forbes AM, Ilett KF. Role of repeated doses of oral activated charcoal in the treatment of acute intoxications. Anaesth Intensive Care 1990; 18: 375-84.

8. Olkkola KT, Neuvonen PJ. Treatment of intoxications using single and repeated doses of oral activated charcoal. J Toxicol Clin Exp 1989; 9: 265-75.

9. Vaughan RS, Mapleson WW, Mushin WW. Prevention of pollution of operating theatres with halothane vapour by adsorption with activated charcoal. BMJ 1973; 1: 727-9.

10. Chang DJ, Choi SH, Choi YS, Min KT. Effect of charcoal filter on the emergence from sevoflurane anesthesia in a semi-closed rebreathing circuit. Yonsei Med J 2011; 52: 668-72.

11. Coleman MA, Coles S, Lytle T, Bennetts FE. Prevention of atmospheric contamination during isoflurane sedation. Clin Intensive Care 1994; 5: 217-20.

12. Kim TW, Nemergut ME. Preparation of modern anesthesia workstations for malignant hyperthermia-susceptible patients: a review of past and present practice. Anesthesiology 2011; 114: 205-12.

13. Gunter JB, Ball J, Than-Win S. Preparation of the Drager Fabius anesthesia machine for the malignant-hyperthermia susceptible patient. Anesth Analg 2008; 107: 1936-45.

14. Birgenheier N, Stoker R, Westenskow D, Orr J. Activated charcoal effectively removes inhaled anesthetics from modern anesthesia machines. Anesth Analg 2011; 112: 1363-70.

15. Enlund M, Wiklund L, Lambert H. A new device to reduce the consumption of a halogenated anaesthetic agent. Anaesthesia 2001; 56: 42932.

16. Berton J, Sargentini C, Nguyen JL, Belii A, Beydon L. AnaConDa reflection filter: bench and patient evaluation of safety and volatile anesthetic conservation. Anesth Analg 2007; 104: 130-4.

17. Perhag L, Reinstrup P, Thomasson R, Werner O. The Reflector: a new method for saving anaesthetic vapours. Br J Anaesth 2000; 85: 482-6.

18. Doyle DJ, Byrick R, Filipovic D, Cashin F. Silica zeolite scavenging of exhaled isoflurane: a preliminary report. Can J Anaesth 2002; 49: 799804.

19. Jänchen J, Brückner JB, Stach H. Adsorption of desflurane from the scavenging system during high-flow and minimal-flow anaesthesia by 
zeolites. Eur J Anaesthesiol 1998; 15: 324-9.

20. Martin J. Inhaled anesthetics: metabolism and toxicity. Miller's Anesthesia. 7th ed. Churchill Livingstone Elsevier, Philadelphia. 2010, pp 633-66.

21. Dupressoir CA. A practical apparatus for measuring average exposure of operating theatre personnel to halothane. Anaesth Intensive Care 1975; 3: 345-7.

22. Ryan SM, Nielsen CJ. Global warming potential of inhaled anesthetics: application to clinical use. Anesth Analg 2010; 111: 92-8.

23. Sulbaek Andersen MP, Sander SP, Nielsen OJ, Wagner DS, Sanford TJ Jr, Wallington TJ. Inhalation anaesthetics and climate change. Br J Anaesth 2010; 105: 760-6. 